\title{
Working memory for stimulus-context associations in rats
}

\author{
ROBERT M. J. DEACON and J. NICHOLAS P. RAWLINS \\ University of Oxford, Oxford, England
}

\begin{abstract}
On each of 10 trials a day, rats were allowed to sample two different target stimuli, each presented in one of two separate and distinctive contexts. One context was arbitrarily and permanently designated as positive for each rat. At a later test in a third apparatus, rats were rewarded for choosing the stimulus that had been encountered in their designated positive context. A pool of 20 different stimuli was freshly randomized between the contexts every day, individually for each rat. About half of the rats demonstrated statistically significant performance under the most stringently controlled conditions, showing that they had a working memory (albeit limited) for contexts no longer physically present. Some possible mechanisms underlying this ability are discussed.
\end{abstract}

The ability of rats to perform both nonspatial and spatial working memory tasks is well known (Aggleton, 1985; Olton \& Samuelson, 1976). In these tasks, the physical stimuli used during the training stage, which provide the essential cues for correct performance, are generally also present at testing. This permits the use of relatively simple cognitive mechanisms, such as associative memory for reward, and, particularly, a process of perceptual fluency that may underly recognition memory. The nonspatial delayed-matching- or nonmatching-to-sample task can be performed by perceiving the test samples and basing a choice on the speed of perceptual processing. To choose the correct nonmatching stimulus, approach the stimulus requiring more processing; conversely, for matching, approach that requiring less processing. Tests of serial order can rely on subtle differences in perceptual fluency depending on how recently the stimulus was seen; it has been shown that increasing stimulus presentation time at the sampling stage is operationally equivalent to having seen the stimulus more recently if the test is not specifically designed to avoid this mechanism (MacDonald, 1993): Probes showed that pigeons were responding primarily on the basis of the strength of the memory trace rather than recalling episodic details of the order of items, as performance was initially disrupted by varying the sample duration (MacDonald, 1993). Note that this does not show that pigeons are incapable of serial episodic recall when appropriate training is given (MacDonald, 1993; Terrace, Chen, \& Jaswal, 1996). Perceptual fluency can also explain the shape of the serial position curve for lists of stimuli in rats. At short retention intervals, the

This work was supported by a Medical Research Council grant to the Brain and Behaviour Research Centre, Oxford University. Correspondence should be addressed to R. M. J. Deacon, Department of Experimental Psychology, University of Oxford, South Parks Rd., Oxford OX13UD, England (email: robert.deacon@psy.ox.ac.uk). most recently seen items are processed more quickly than are earlier ones and are consequently remembered better, giving rise to a recency effect. As the retention period increases, the relative recency of all list items tends to a constant value (or Weber fraction) and the serial position curve becomes flat (Deacon \& Rawlins, 1995).

Likewise, in the simple version of the radial arm maze spatial working memory task in which all arms are initially baited, a rat runs up a baited arm, viewing the extramaze cues, and consumes the reward. Later, faced with the decision of whether to run the same arm again, it views the configuration of extramaze cues associated with this arm and compares this with the cue configuration of another arm; if the latter is processed more slowly, it is the correct one. In this example, many more mechanisms may contribute to successful performance, such as associative memory of reward with the first arm and operation of a winshift strategy. However, as with the nonspatial tasks, all the physical stimuli essential to the procedure (maze, room cues) are physically present at testing.

In human memory, a simple distinction between recognition memory and recall is that, in the former, a stimulus is physically presented to the subject and a judgment of familiarity is required. Thus, this can be performed by a simple process of perceptual fluency. In recall, however, images/events that are related only by association to a target stimulus (or question) are retrieved from memory; these images/events are no longer within the external perceptual field of the subject.

Animal tests that rely on working associative memory for stimuli no longer physically present at testing may therefore be useful in modeling higher order human cognitive capacities (e.g., episodic memory) or at least may require more complex cognitive mechanisms than in the above examples of animal working memory and thus be of interest in their own right. In the present study, rats were trained to remember where they had recently encountered a stimulus object. In the sampling phase of each trial, a rat was successively presented with two target stimuli. 
One stimulus was in an apparatus arbitrarily designated as a positive context (i.e., to be rewarded later by association), whereas the other stimulus was presented in another context designated negative. At the retention test in a third apparatus, the rat was required to choose between the target stimuli according to which context they had been encountered in; only the stimulus that had previously been encountered in the positive context on that particular day was rewarded. Each day the stimuli were randomly allocated to either the positive or the negative context. Unlike in many tests of animal learning and memory, the crucial stimuli - the contexts in which the targets were originally sampled--were no longer physically present at test and could only be accessed via an internal representation, a stimulus-context association unique to that trial. The task is similar to paired associate learning or symbolic matching, which involve the presentation at retention of stimuli that are only conditionally related to the to-be-remembered samples, but these latter are incrementally learned tasks, the associative component of which does not rely on working memory.

\section{METHOD}

\section{Subjects}

Ten male Dark Agouti rats (Olac, Bicester, U.K.), approximately 2 months old and weighing $150-170 \mathrm{~g}$ on arrival, were housed in pairs in wire cages in a temperature-controlled room on a 12:12-h light:dark cycle (lights on at $0700 \mathrm{~h}$ ). All testing took place during the light phase in an adjacent room. The rats had constant access to water. Each pair was fed 16-28 g (according to individual rat weight and spillage) of laboratory chow per day after the testing session. This is a mild deprivation schedule, just sufficient to provide good motivation.

\section{Apparatus}

Three different pieces of apparatus, which will be referred to as Context 1, Context 2, and the Y-maze, were used to contain the target stimuli. Contexts 1 and 2 served as sampling contexts during the first stage of a trial, and the Y-maze was used for choice tests.

Context 1 was an open elevated $(47 \mathrm{~cm} \mathrm{high)}$ wooden table covered with plastic laminate. A start area $(18 \times 12 \mathrm{~cm})$ broadened into an approach/goal area $40 \mathrm{~cm}$ wide at its furthest point, $41 \mathrm{~cm}$ from the start. A $2-\mathrm{cm}$ beading surrounded both approach/goal and start areas. The far end of the approach/goal area was centrally divided by a low plywood wall $4 \mathrm{~cm}$ high, $15 \mathrm{~cm}$ long, forming two goal areas; only the goal area on the rat's right was used in the results reported here.

Context 2 was enclosed, with sheet metal walls and floor and a clear Plexiglas lid. A start area $19 \times 9 \mathrm{~cm}$ broadened into an approach area $25 \mathrm{~cm}$ wide at its furthest point, $15 \mathrm{~cm}$ from the start. Beyond the approach area were two goal areas $12 \mathrm{~cm}$ wide separated by a wall of clear Plexiglas extending to the lid, $20 \mathrm{~cm}$ high. Only the goal area on the rat's left was used in the results reported here; the other side was blocked by a sheet metal door. The approach and goal areas were separated by a wooden block threshold $3 \mathrm{~cm}$ high, $3 \mathrm{~cm}$ broad.

The Y-maze was also made of sheet metal with a clear Plexiglas lid. The start arm was separated by a metal central trefoil guillotine door from the two choice arms. Each arm measured $15 \times$ $13 \times 20 \mathrm{~cm}$. At the end of each choice arm was a guillotine door and a goal area. Doors were manually operated by a string-andpulley system.
Twenty distinctive goal boxes could be placed in the goal area of each maze. Each box measured $8 \times 12 \times 16 \mathrm{~cm}$ and was open on one $12-\mathrm{cm}$ side, with the floor extending a further $9 \mathrm{~cm}$; this end faced the maze center. These boxes were made of plywood painted different colors, and each contained an individual array of small junk objects, such as clothes pegs, nuts and bolts, toy animals, and so on, glued to the floor and walls (Aggleton, 1985). Each box had a matching duplicate. The duplicates looked identical to the human eye but, presumably, may have smelled slightly different to the rats, depending on the box materials (nominally identical) and traces of previous rats. (It is not feasible to deodorize this type of stimulus box between each rat, and one cannot prove that a rat cannot detect an odor.) In both Context 2 and the Y-maze, the goal areas were illuminated with three 2.8 -W bulbs to supplement the normal fluorescent room lighting. A hole in the lid above each goal area allowed food pellets (Noyes, $45 \mathrm{mg}$ ) to be dropped into the goal boxes after the rat had entered. For Context 1 , food pellets were preplaced in or close to the goal box.

Contexts 1 and 2 were set $1 \mathrm{~m}$ apart on a bench on a side wall, with the Y-maze $1.5 \mathrm{~m}$ away in the center of the laboratory. The walls around Context 1 were covered with distinctive junk objects.

\section{Procedure}

The rats were progressively habituated to handling and the apparatus, with blank stimulus boxes (unpainted, containing no objects) placed in the goal areas of each context/maze, where food pellets could be delivered.

For each rat, either Context 1 or Context 2 was permanently designated as positive. Any stimulus box seen in the positive context would be rewarded when it was later presented during the test phase, conducted in the Y-maze. Correspondingly, presentation of a box in the negative context signaled that it would not be rewarded later in the Y-maze. Contexts 1 and 2 were distinguishable by their different construction, position in the room, position of goal area (right or left of the start/approach area), mode of pellet delivery (preplaced on the floor or delivered after entry from above), and whether or not there was a threshold in front of the goal box.

Formal training began when all rats were running the mazes freely and eating pellets in the boxes. Over a period of 5 months, the rats were trained with several variations in procedure. These included presenting lists of stimuli, variations in the number of trials per day and reinforcements per trial and use of correction trials. Because some of these training procedures may not have been optimal, and for reasons of space, they will not be described here. ${ }^{1}$

The final protocol was as follows. On each trial, the rat was placed first in one context to sample a goalbox where it received two food pellets, then in the other context to sample another box (also containing two pellets). The pellets were given simply to facilitate stimulus sampling and were not contingent on any aspect of the rat's behavior. Order of context presentation was separately randomized for each day. At testing, the rat was put into the Y-maze to choose between these two boxes. Choice of the correct box (that visited in the arbitrarily positive context) was rewarded with five pellets. Sampling each box normally took $30 \mathrm{sec}$ or less; intercontext time was approximately $5 \mathrm{sec}$. Testing occurred approximately $5 \mathrm{sec}$ after the rat consumed the pellets in the second context visited. Ten trials per day were run, each separated from the next by at least $30 \mathrm{~min}$. A correction procedure was used. If the rat entered the box from the negative context at testing, it was returned to the positive context with the appropriate box in place (i.e., the one unentered in the Y-maze). Up to two further choices and corrections were given; then, if necessary, on the third correction trial, only the correct box was available, the guillotine door to the incorrect one being shut.

After 5 months of training, it was apparent that a crucial procedural variable was whether the boxes used at testing were the actual ones originally sampled in the context mazes or were dupli- 
cate versions (which look identical to the human eye). (These will be referred to simply as original and duplicate boxes, respectively.) All rats performed well on original-box trials, but performance on duplicate-box trials was poor for many rats. The main results presented here were obtained during 27 days of the final phase of training, using both original- and duplicate-box trials. Five trials of each type were given daily, with a different order each day.

Two further supplementary experiments were performed to investigate the original-box-duplicate-box difference.

Olfactory probes. If the rats were choosing between the test boxes according to whether a box smelled of the sample context it had recently been placed in, this would explain why performance was better on original box trials as compared to duplicates. All rats were therefore tested in the Y-maze on boxes that had been placed in the sample contexts for $90 \mathrm{sec}$, which was slightly longer than the usual length of time it took to run the sample phase. The rats themselves were not given sample runs, only choice trials. Ten trials a day were run for 3 days.

Stimulus salience probe. The stimulus salience probe was used to determine whether rats predominantly used positive or negative box/context cues. At testing, one of the boxes was an original and the other one was a duplicate of that presented in the other sample context. Thus, on each day, for five randomly selected trials, the original box had been presented in the positive context, whereas, on the other five trials, the original box came from the negative context. Eight days of this semiduplicate condition were preceded and followed by 2 days of testing using only original boxes to provide a baseline for comparison. Thus, there were three conditions of testing in the Y-maze: (1) both boxes were originals; (2) the box from the negative sample context was an original, that from the positive a duplicate; (3) the box from the negative sample context was a duplicate, that from the positive an original.

\section{RESULTS}

One-sample, two-tailed $t$ tests showed that group mean performance during the last 27 days of training was significantly better than chance for both original and duplicate boxes. However, performance was higher on originalbox trials than on duplicate-box trials (Table 1).

When data from individual rats were examined, all showed performance significantly better than chance on trials with original boxes [minimum $t(26)=4.52, p<$

Table 1

Group Mean and $S E M$ Values and One-Sample Two-Tailed $t$ Tests Comparing Performance Against Chance $(d f=9)$ for the Final 27 Days of Training With Duplicate and Original Test Boxes and for the 12 Days on Which (1) Both Test Boxes Were Those Originally Sampled, (2) the Originally Sampled Positive Box Was Replaced by a Duplicate, or (3) the Originally Sampled Negative Box Was Replaced by a Duplicate

\begin{tabular}{|c|c|c|c|c|}
\hline \multirow{2}{*}{$\begin{array}{c}\text { Box Type } \\
\text { at Test }\end{array}$} & \multicolumn{2}{|c|}{ Percent Correct } & \multirow[b]{2}{*}{$t$} & \multirow[b]{2}{*}{$P$} \\
\hline & $M$ & $S E M$ & & \\
\hline \multicolumn{5}{|c|}{ Final 27 Days of Training } \\
\hline $\begin{array}{l}\text { Original } \\
\text { Duplicate }\end{array}$ & $\begin{array}{l}79.9 \\
59.0\end{array}$ & 3.5 & $\begin{array}{l}8.62 \\
4.51\end{array}$ & $\begin{array}{l}<.001 \\
<.005\end{array}$ \\
\hline
\end{tabular}

12 Days With Both Originally Sampled Test Boxes or One Originally Sampled Test Box

\begin{tabular}{lllll} 
Original & 79.0 & 4.9 & 5.98 & $<.001$ \\
Duplicate positive & 78.3 & 3.9 & 7.29 & $<.001$ \\
Duplicate negative & 63.0 & 3.7 & 3.47 & $<.01$ \\
\hline
\end{tabular}

Table 2

Results for the Rats Whose Performance Was Significantly Greater Than Chance on the Final 27 Days of the Episodic Memory Task With Duplicate Test Boxes; One-Sample, Two-Tailed $t$ Tests $(d f=26)$ Are Shown

\begin{tabular}{ccccc}
\hline & \multicolumn{2}{c}{ Percent Correct } & & \\
\cline { 2 - 5 } Rat & $M$ & $S E M$ & $t$ & $p$ \\
\hline 1 & 65.2 & 3.9 & 3.86 & $<.001$ \\
2 & 62.2 & 3.9 & 3.14 & $<.005$ \\
3 & 70.4 & 4.0 & 5.03 & $<.001$ \\
7 & 63.7 & 3.4 & 4.05 & $<.001$ \\
9 & 60.7 & 3.9 & 2.74 & $<.025$ \\
\hline
\end{tabular}

$.001]$. For duplicate boxes, 5 of the 10 rats scored significantly better than chance (Table 2). The order in which the stimuli/contexts were sampled did not have a significant effect (and was counterbalanced anyway). A repeated measures two-way analysis of variance (ANOVA) showed that no effect of order was present $[F(1,48)=$ $1.76, p=.19]$, nor was there an interaction between the duplicate/original condition and sample order $[F(1,48)=$ $1.76, p=.19]$.

The group as a whole scored significantly above chance during the olfactory probes [group mean $=64.6 \%, t(9)=$ $5.85, p<.001]$. However, there was no correlation between the olfactory probe scores and those from the original component of final training $(r=-.178, p=.623)$.

In the final semiduplicate condition, performance remained relatively unaffected when the box from the positive context was a duplicate at testing (Table 1). When the negative-context box was a duplicate, however, performance dropped by $15.3 \%$. An ANOVA showed that this effect of box type was significant $[F(2,18)=10.042, p<$ $.005]$. There was also a nonsignificant tendency for the rats assigned Context 1 as positive to perform better than those assigned to Context $2[F(1,8)=4.87, p<.1]$.

\section{DISCUSSION}

All rats were able to discriminate the test boxes according to the contexts in which they had been sampled on that particular day. Performance was better when the test box was the original one sampled. When a duplicate box was used, 5 of the 10 rats still performed significantly better than chance and so appeared to have a memory of a box-context association. The 3 days of olfactory probes using unsampled boxes suggested that most rats could make some use of an alternative, simpler strategy - that of selecting boxes according to olfactory cues from the context in which the boxes had recently been placed. The better performance on original boxes than on duplicate boxes may partly reflect an ability to use this strategy. The lack of a correlation between the rats' performance on original boxes and the scent-probe days indicates that this cannot account for more than a small part of the original-duplicate difference. However, the training procedure itself and the relative salience to the rats of cues from different sensory modalities may provide a better 
explanation. Since olfactory cues are probably more salient to rats than are tactile/visual cues (Nigrosh, Slotnick, \& Nevin, 1975), a nominally duplicate box at testing might be perceived (smelled) as very different from that originally sampled, although it looked the same. Since the task rule during training was "at testing, enter the box recently sampled in the positive context," good performance on duplicate boxes would require some modification of this basic rule, since original boxes were used throughout the earlier training. Olfactory stimuli from the boxes themselves may have overshadowed box cues from other modalities. If we had used duplicate boxes at all stages of training, performance on these might have been as good as with original boxes. Alternatively, the rats might never have learned the task if the olfactory component of the box, which could have served as an intermediate cue for the correct response, was absent.

Better performance on original boxes might also have resulted from the rats scent marking the boxes at the sampling stage. Active scent marking was not observed, however, and this explanation presupposes that they "knew" which context was positive and which negative. Presumably, passive scent cues would not distinguish the negative and positive boxes, since both had been entered at sampling.

The rats seemed to predominantly use cues from the box sampled in the negative context: Performance was markedly worse when this box was a duplicate (see Table 1). Whether discriminations are performed by learning to approach the positive stimulus or to avoid the negative appears to depend on the particular task used (Mackintosh, 1974).

In summary, the results indicate that rats probably perform the task using a variety of strategies and perceptual modalities. While a (limited) component of performance may be due to a first-order association between residual context odor and reward at test (which by definition is "artifactual" in the present experiment, not source/episodic memory), this cannot fully account for the magnitude of the scores on original boxes. When this component was dissected out by using duplicate boxes, some rats still demonstrated a working memory ability, limited but nonetheless statistically significant, to remember in which context they had encountered a stimulus. This demonstrates that rats may have a capacity analogous to source, episodic, or prospective memory, but the results of the present experiment suggest that this may be limited.

This memory task in rats can be compared to other tasks considered to depend on episodic memory. In one experiment, a monkey was first rewarded for choosing any one of three simultaneously presented stimuli. Later, the chosen stimulus and one of the untouched (but previously seen) stimuli were presented; only choice of the latter was rewarded. This task cannot be solved by recognition memory alone (unlike the superficially similar delayed nonmatching task). The monkey must remember not only what it saw, but what it did. In contrast, the second task was exactly the same as the first, except that the previously touched object was now paired with a com- pletely novel, unseen object at test. This test could be performed by simple recognition processes (e.g., perceptual fluency) and, unlike the first, was unaffected by a lesion of the mid-dorsolateral frontal cortex (Petrides, 1991).

A recently developed memory task requires monkeys to concurrently discriminate many positive and negative visual stimuli, each pair being presented within a unique complex visual background on a touch screen monitor (Gaffan, 1994). Lesions of brain regions crucial to human episodic memory impair performance of this task, though not completely. Successful performance depends on an episodic memory of touching a discrete part of a stimulus array, which is then associated with reinforcement. Similarly, although the first task of Petrides is a working memory paradigm, the monkey must remember which component of the stimulus array it touched. In our episodic working memory task, the rat must remember a unique conjunction of external stimuli (box and context) rather than a juxtaposition of its own actions and an object within grasping space.

Detailed work would be required to determine if the present rat task depends on episodic memory as believed to occur in primates. If the initially arbitrarily designated negative context acquired real negative valence during the training procedure, prospective memory could guide test choices. The rats might assign a higher order negative valence to boxes entered in the negative context. Accurate test choices could then be made simply by avoiding boxes with such negative valence when seen later in the Y-maze. An anonymous reviewer has noted that, on this account, our paradigm would be similar to a directed forgetting experiment. In this, an animal is frequently given a "forget" cue after a sample (typically in a delayed matching task), which signals that that sample will not be tested. Occasional probe trials, where the sample is tested after a "forget" cue, reveal lower performance than do standard trials. Our negative context could thus have acted similarly to a "forget" cue, or the positive context as a "remember" cue. This would overcome one of the problems associated with the interpretation of directed forgetting experiments, where impaired performance after "forget" cues may be due to an expectation of nonreinforcement, causing frustration and response biases rather than forgetting (Zentall, Roper, \& Sherburne, 1995). Presenting both "to-be-remembered" and "to-beforgotten" material within a trial, as in a development of our paradigm, might help to overcome this problem. Our paradigm is not exactly parallel to directed forgetting the negative context signals "avoid this stimulus at test" rather than "this stimulus will not be presented for a test." However, comparing the paradigms might be informative.

The development of episodic memory may be partly dependent on perceptual factors. Olfactory stimuli are long lasting relative to the component visual and auditory stimuli of an event. Macrosmatic animals may be less dependent on episodic memory than animals with highly developed visual systems, such as primates, especially those with complex social lives (Byrne \& Whiten, 1988). In the dynamic social environment of apes and monkeys, 
where there are strong individual relationships that may suddenly change according to events in the life of the group, a selective advantage would result from remembering exactly who did what and to whom.

Prospective memory is compromised in amnesic and dementing disorders (Huppert \& Beardsall, 1993). Source amnesia has been shown to be independent from recall and recognition in amnesic states and may represent a specific fact-context association deficit (Dywan, Segalowitz, Henderson, \& Jacoby, 1993; Shimamura \& Squire, 1991). The further development of animal memory models such as that reported here could facilitate research into these areas.

\section{REFERENCES}

Aggleton, J. P. (1985). One-trial object recognition by rats. Quarterly Journal of Experimental Psychology, 37B, 279-294.

BYRNE, R. W., \& WHITEN, A. (EDS.) (1988). Machiavellian intelligence: Social expertise and the evolution of intellect in monkeys, apes, and humans. Oxford: Oxford University Press, Clarendon Press.

Deacon, R. M. J., \& Rawlins, J. N. P. (1995). Serial position effects and duration of memory for nonspatial stimuli in rats. Journal of Experimental Psychology: Animal Behavior Processes, 21, 285-292.

Drwan, J., Segalowitz, S. J., Henderson, D., \& Jacoby, L. (1993). Memory for source after traumatic brain injury. Brain \& Cognition, 21, 20-43

GafFan, D. (1994). Scene-specific memory for objects: A model of episodic memory impairment in monkeys with fornix transection. Journal of Cognitive Neuroscience, 6, 305-320.

HupPert, F. A., \& BeARdSAll, L. (1993). Prospective memory impair- ment as an early indicator of dementia. Journal of Clinical \& Experimental Neuropsychology, 15, 805-821.

MacDonald, S. E. (1993). Delayed matching-to-successive-samples in pigeons: Short-term memory for item and order information. $A n$ imal Learning \& Behavior, 21, 59-67.

MACKINTOSH, N. J. (1974). The psychology of animal learning. London: Academic Press.

Nigrosh, B. J., Slotnick, B. M., \& Nevin, J. A. (1975). Olfactory discrimination, reversal learning, and stimulus control in rats. Journal of Comparative \& Physiological Psychology, 89, 285-294.

Olton, D. S., \& Samuelson, R. J. (1976). Remembrance of places passed: Spatial memory in rats. Journal of Experimental Psychology: Animal Behavior Processes, 2, 97-116.

Petrides, M. (1991). Monitoring of selections of visual stimuli and the primate frontal cortex. Proceedings of the Roval Society of London: Series B, 246, 293-298.

Shimamura, A. P., \& Squire, L. R. (1991). The relationship between fact and source memory: Findings from amnesic patients and normal subjects. Psychobiology, 19, 1-10.

Terrace, H. S., Chen, S., \& Jaswal, V. (1996). Recall of three-item sequences by pigeons. Animal Learning \& Behavior, 24, 193-205.

Zentall, T. R., Roper, K. L., \& Sherburne, L. M. (1995). Most directed forgetting in pigeons can be attributed to the absence of reinforcement on forget trials during training or to other procedural artifacts. Journal of the Experimental Analysis of Behavior, 63, $127-137$.

\section{NOTE}

1. Details of initial training will be supplied on request.

(Manuscript received May 30, 1996;

revision accepted for publication November 29, 1996.) 